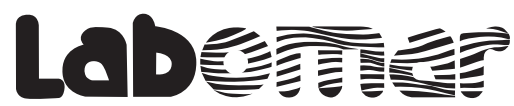

Arquivos de Ciências do Mar

\section{COMUNIDADE ZOOPLANCTÔNICA DA PLATAFORMA CONTINENTAL DE SALVADOR, BAHIA}

\section{Zooplankton community the continental shelf of Salvador, Bahia}

\author{
Laura Rodrigues da Conceição ${ }^{1,2}$, Christiane Sampaio de Souza ${ }^{3}$, \\ Paulo de Oliveira Mafalda Junior 4 \\ ${ }^{1}$ Bacharel em Oceanografia, Laboratório de Plâncton, Instituto de Biologia, Universidade Federal da Bahia. E-mail: laura_faro@hotmail.com \\ ${ }^{2}$ Mestranda do Programa de Pós-graduação em Oceanografia PPGO/UFPE, Universidade Federal de Pernambuco. E-mail: laura_faro@ \\ hotmail.com \\ ${ }^{3}$ Laboratório de Plâncton, Instituto de Biologia, Universidade Federal da Bahia. E-mail: chsampaio@ig.com.br \\ ${ }^{4}$ Laboratório de Plâncton, Instituto de Biologia, Universidade Federal da Bahia. E-mail: paulomafa@hotmail.com
}

\begin{abstract}
RESUMO
Para a região oceânica do Atlântico Sul Tropical, pesquisas voltadas para a comunidade zooplanctônica, seu comportamento e distribuição, bem como o aparato bibliográfico, ainda são escassas. Inserido nesse contexto, o objetivo deste trabalho foi identificar a composição e densidade do zooplâncton além de avaliar a sua variabilidade espacial e temporal através da plataforma continental de Salvador. As coletas de água e zooplâncton ocorreram bimestralmente, entre abril de 2013 e janeiro de 2014, em seis cruzeiros oceanográficos com quatro estações amostrais posicionadas entre a costa e o talude continental de Salvador. Em todo o período de estudo foi registrada apenas a presença da massa de Água Tropical. A densidade total do zooplâncton apresentou variabilidade temporal, porém não apresentou variabilidade espacial. A composição da comunidade zooplanctônica foi característica de ambiente marinho tropical oligotrófico, destacando-se: copépodes, moluscos, quetognatos, cndiários e larváceos. A abundância de grupos holoplanctônicos predominou sobre os meroplanctônicos. A variabilidade na composição foi explicada pelo gradiente oceanográfico gerado pela massa de água. A análise multivariada mostrou uma variabilidade temporal e espacial da densidade do zooplâncton em função das variáveis oceanográficas, mostrando uma forte influência de uma massa de Água Tropical, extremamente oligotrófica na comunidade zooplanctônica.
\end{abstract}

Palavras-chave: zooplâncton, densidade, plataforma continental, Salvador.

Recebido: 15 jun 2016

Aceito: 21 jan 2017

Publicado online: 31 mai 2017 


\section{ABSTRACT}

For oceanic region of the Tropical South Atlantic, research aimed at a zooplankton community, its behavior and distribution, as well as the bibliographic apparatus, are still scarce. In this context, the present study aimed to identify the composition and density of zooplankton and evaluate your spatial and temporal variability of through Salvador continental shelf. Sampling of water and zooplankton were held bimonthly between April 2013 and January 2014, in six oceanographic cruises with four sampling stations positioned between the coast and the continental slope. Throughout the study period was recorded only the presence of the mass of Tropical Water. The total density of zooplankton showed seasonal variability, but showed no spatial variability. The composition of the zooplankton community of Salvador continental shelf was characteristic of oligotrophic tropical marine environment. Abundance of holoplanktonics groups predominated over meroplanktonic. The variability in the composition was explained by the oceanographic gradient generated by the mass of water. Multivariate analysis showed a temporal and spatial variability in the density of zooplankton in relation to oceanographic variables, which shows a strong influence of a mass of tropical water, extremely oligotrophic in the zooplankton community.

Key words: zooplankton, density, continental shelf, Salvador

\section{INTRODUÇÃO}

O zooplâncton desempenha um papel chave na teia trófica, tanto no ambiente marinho quanto no ambiente limníco (Kiørboe, 2008). O conhecimento da estrutura e funcionamento das comunidades zooplanctônicas é, portanto, de grande importância para uma melhor compreensão dos ecossistemas aquáticos. No ambiente marinho, esses organismos apresentam uma diversidade significativa de estratégias ecológicas, padrões de dominância e distribuição além de influenciarem e refletirem as condições do ecossistema no qual estão inseridos (Litchman et al., 2013).

A comunidade zooplanctônica como um todo é desigualmente distribuída, apresentando-se de forma agrupada no ambiente marinho. Além disso, ela é frequentemente estruturada em assembleias tendo uma estreita relação com as características ambientais. Assim, a distribuição das massas de água e correntes bem como fatores químicos, físicos e biológicos que caracterizam a água e sua qualidade, constituem a base para a interpretação dos padrões de distribuição desses organismos (Berasategui et al., 2006).

Os primeiros estudos com zooplâncton marinho na Bahia foram realizados por Paranaguá (1963) onde foi avaliada a distribuição do zooplâncton desde a plataforma do Ceará até a Baía de Todos os Santos, apontando a presença de uma estrutura trófica característica de ambiente oligotrófico. Durante o programa REVIZEE foram realizados levantamentos que implicaram na caracterização da distribuição espacial e temporal da biomassa secundária entre a plataforma continental e a zona oceânica a partir de Salvador (Bonecker et al., 2006; Mafalda Jr. et al., 2009b). Na plataforma continental de Salvador a distribuição do zooplâncton foi caracterizada por baixas densidades (Mafalda Jr. et al., 2009b) e um gradiente de biomassa na região central da plataforma central da Zona Econômica Exclusiva (ZEE), entre Salvador e o cabo de São Tomé (RJ), orientado no sentido oceano-plataforma continental, foi constatado (Bonecker et al., 2006). Resultados de peso úmido e densidade do mesozooplâncton obtidos na plataforma de interna de Salvador mostraram valores máximos de 
10 g. $100 \mathrm{~m}^{-3}$ e 8000 org. $\mathrm{m}^{-3}$ respectivamente, e valores menores em águas oceânicas com a predominância de copépodes, larvaceas e ostracodos (Bonecker et al., 2006). Valores mais elevados de biomassa secundária foram registrados entre Aracajú e Salvador e no norte da Baía de todos os Santos.

Uma análise da comunidade zooplanctônica de cinco domínios ecológicos diferentes em águas superficiais compreendendo a plataforma continental e região oceânica em torno de $37^{\circ} \mathrm{S}$ incluindo a confluência Brasil-Malvinas foi proposta e seus resultados mostraram Copepoda como grupo dominante durante todo o estudo e a fração do mesozooplâncton contribuiu com $40 \%$ da densidade total do zooplâncton, com picos de biomassa em torno de 50\% (Thompson et al., 2014). Variáveis oceanográficas representadas pela temperatura da superfície do mar, salinidade e clorofila- $a$ foram as variáveis ambientais que melhor explicaram a tendência distribuição de zooplâncton (Thompson et al., 2014).

O objetivo deste trabalho foi descrever a composição da comunidade zooplanctônica bem como sua densidade e também caracterizar a estrutura oceanográfica da massa de água além de analisar a distribuição espacial e temporal do zooplâncton em função das características oceanográficas através da plataforma continental de Salvador, numa transeção perpendicular à costa.

\section{MATERIAL E MÉTODOS}

\section{Área de estudo}

A plataforma continental de Salvador está compreendida nas coordenadas $13^{\circ} 01^{\prime} 00^{\prime \prime} \mathrm{S}$ e $13^{\circ} 08^{\prime} 5400^{\prime \prime} \mathrm{S}$ e $38^{\circ} 29^{\prime} 5200^{\prime \prime} \mathrm{W}$ e $38^{\circ} 29^{\prime} 4400^{\prime \prime} W$ (Figura 1). É considerada a mais estreita de toda a costa brasileira medindo entre 8 (Knoppers et al., 1999; Pereira, 2009) e $11 \mathrm{~km}$ (Cooke et al., 2007) com a quebra da plataforma ocorrendo entre 50 e 60 $\mathrm{m}$ de profundidade (Knoppers et al., 1999; Pereira, 2009). Assim como na Baía do Todos os Santos (feição compreendida nas bordas da cidade de Salvador), que tem uma vazão estuarina limitada devido ao baixo aporte fluvial (Hatje \& Andrade, 2009) os rios que banham a plataforma continental de Salvador possuem uma vazão média que não chega a 2 $\mathrm{m}^{3} \cdot \mathrm{s}^{-1}$ (INEMA, 2008). Sendo assim, a área estudada caracteriza-se como uma região típica

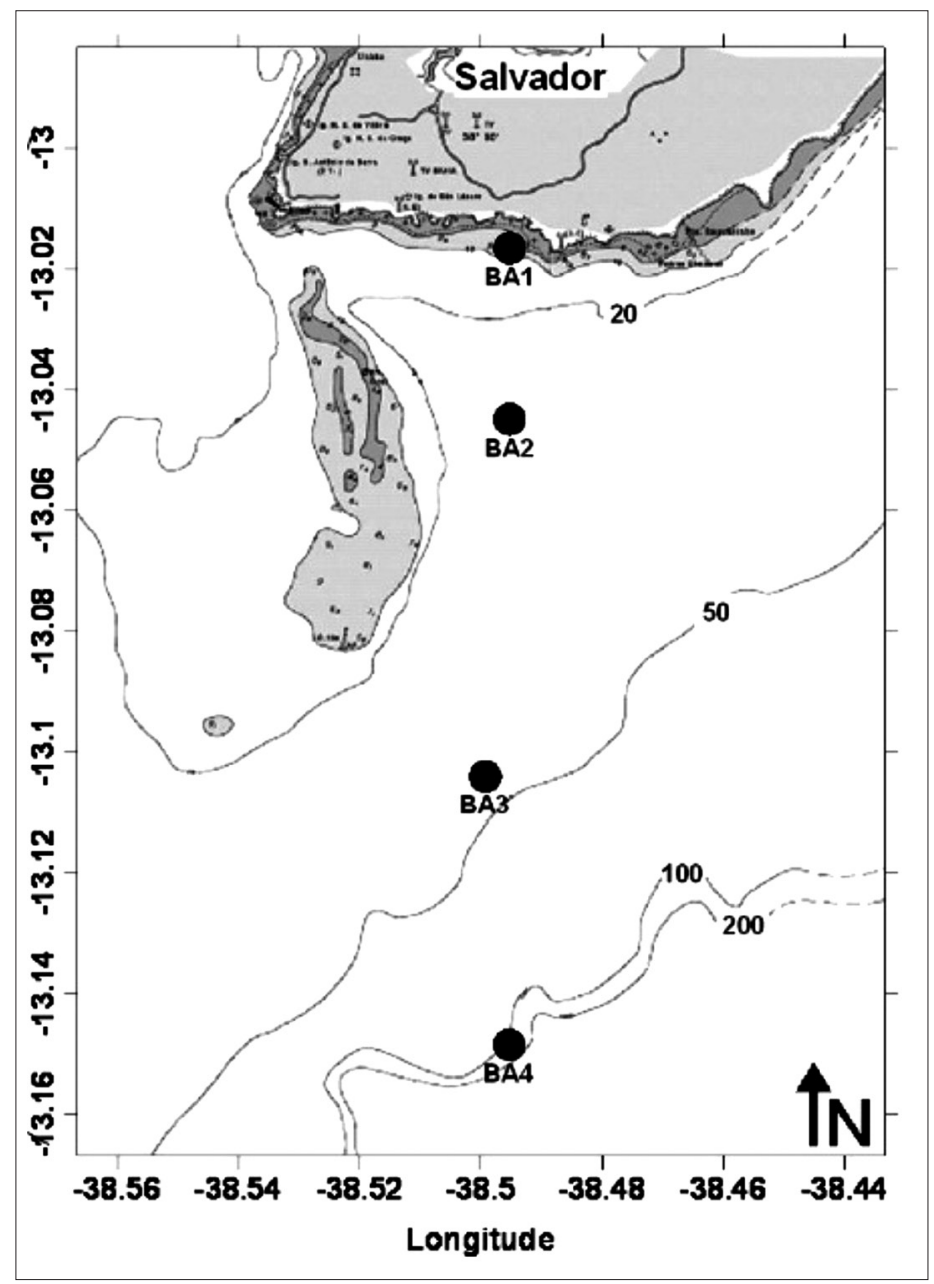

Figura 1 - Área de estudo e localização das estações de amostragem na plataforma continental de Salvador, Bahia. 
da plataforma continental do Nordeste, com águas quentes e oligotróficas (Ekau \& Knoppers, 1999; Paredes, 1991).

Além disso, a área estudada está sob influência da Corrente do Brasil, sendo esta responsável por transportar Água Tropical (AT) com salinidade entre 36-38 e temperatura entre $24-26^{\circ} \mathrm{C}$ (Garfield, 1990). O clima da região é classificado como tropical e úmido e, além disso, comumente é registrado um período chuvoso entre março e julho sendo este, responsável por $60 \%$ da precipitação anual e um período seco entre agosto e fevereiro (Pereira \& Lessa, 2009).

\section{Amostragem}

As coletas de água e zooplâncton foram realizadas dentro do contexto do projeto INCTAmbTropic, bimestralmente, entre abril de 2013 e janeiro de 2014 em seis cruzeiros oceanográficos com quatro estações amostrais (BA1, BA2, BA3 e BA4) posicionadas entre a costa e o talude continental de Salvador (Figura 1). As amostras de água foram coletadas com garrafa do tipo Van Dorn (2,L) para análise de oxigênio dissolvido através do Método de Wincler (Apha, 1992). A amostragem do zooplâncton foi realizada através de arrastos horizontais com rede cônica de malha de $300 \mu \mathrm{m}$, com duração de cinco minutos e com um fluxômetro mecânico (Hydro-bios) acoplado. Para a obtenção de dados de temperatura $\left({ }^{\circ} \mathrm{C}\right)$ e salinidade da água do mar foi utilizado Castway CTD (Condutivity, Temperature and Depth). Após coletadas as amostras foram transferidas para frascos plásticos e fixadas a bordo em solução formaldeído a $4 \%$.

As amostras foram submetidas à triagem, identificação, contagem e processamento de dados. As análises empregaram microscópio estereoscópico (lupa) a partir de um volume de amostra padronizado $(300 \mathrm{ml})$ onde foram retiradas alíquotas de $20 \mathrm{ml}$. As amostras foram identificadas a nível de grandes grupos de acordo a literatura proposta (Boltovoskoy, 1981) e submetidas ao cálculo de frequência de ocorrência, estimativa de densidade e cálculo do índice de riqueza de margalef (IRM).

\section{Análise de dados}

Visando investigar a ocorrência de variabilidade temporal e espacial nos dados abióticos (temperatura, salinidade, transparência e oxigênio dissolvido) e bióticos (densidade dos grupos zooplanctônicos) foi aplicado o teste de Shapiro-Wilk para avaliar a sua normalidade. Após verificar a ausência de normalidade foi aplicado o teste não paramétrico de Kruskal-Wallis (Teste de KW), com o objetivo de verificar a existência de variabilidade espacial e temporal utilizando o programa Biostat versão 5.3. A representação da distribuição espacial da densidade total dos principais grupos foi realizada através da elaboração de mapas georreferenciados com intervalos de classe utilizando o programa SURFER FOR WINDOWS da Golden Software Inc. (Keekler, 1995).

A Análise de Correspondência Canônica não tendenciosa (DCCA) foi utilizada para investigar o tamanho do gradiente ambiental. Uma vez que este gradiente foi linear $(1,36)$, foi realizada a Análise de Redundância (RDA) para verificar o principal padrão de variação na composição da associação zooplanctônica em função das variáveis ambientais (Ter Braak, 1986). A matriz de dados biológicos foi formada com base no cálculo da frequência de ocorrência percentual (FR\%) sendo selecionado os grupos zooplanctônicos que ocorreram em todas as campanhas de amostragem $(\mathrm{FO}=100 \%)$. A matriz das variáveis ambientais foi composta por dados de temperatura $\left({ }^{\circ} \mathrm{C}\right)$, salinidade, transparência $(\mathrm{m})$ e oxigênio dissolvido (mg. $\left.\mathrm{L}^{-1}\right)$. As análises DCCA e RDA foram realizadas empregando o pacote estatístico Canoco for Windows versão 4.5 (Ter Braak \& Smilauer, 1998). 


\section{RESULTADOS}

\section{Temperatura}

Durante o período de amostragem a temperatura registrou seu menor valor $\left(25,92^{\circ} \mathrm{C}\right)$ na campanha 4 no ponto BA1 e maior $\left(29,05^{\circ} \mathrm{C}\right)$ na campanha 1 no ponto BA4. (tabela 1$)$. Foi registrada variabilidade temporal $(\mathrm{p}<0,05)$ porém, não foi registrada variabilidade espacial $(\mathrm{p}>0.05)$.

\section{Salinidade}

A salinidade registrou seu menor valor (36) na campanha 2 no ponto BA1 seu maior valor $(39,98)$ na campanha 5 no ponto BA1. Foi registrada variabilidade temporal $(p<0,05)$ e não foi registrada variabilidade espacial $(\mathrm{p}>0,05)$.

A partir dos dados de temperatura e salinidade foi possível registrar a presença da massa de Água

Tabela I - Valores máximos, mínimos, média e desvio padrão calculados para as variáveis abióticas (temperatura, salinidade, oxigênio dissolvido e transparência) na plataforma continental de Salvador, Bahia, no período de abril/2013 a janeiro/ 2014.

\begin{tabular}{c|ccc}
\hline Temperatura $\left({ }^{\circ} \mathrm{C}\right)$ & Máximo & Mínimo & Média e DP \\
Salinidade & 29,05 & 25,92 & $27,11 \pm 0,92$ \\
OD $\left(\mathbf{m g}, \mathbf{L}^{-1}\right)$ & 39,98 & 36 & $37,25 \pm 0,79$ \\
Secchi $(\mathbf{m})$ & 7,60 & 4,90 & $6,10 \pm 0,70$ \\
\hline
\end{tabular}
Tropical, durante todo o período de estudo (Figura 2).

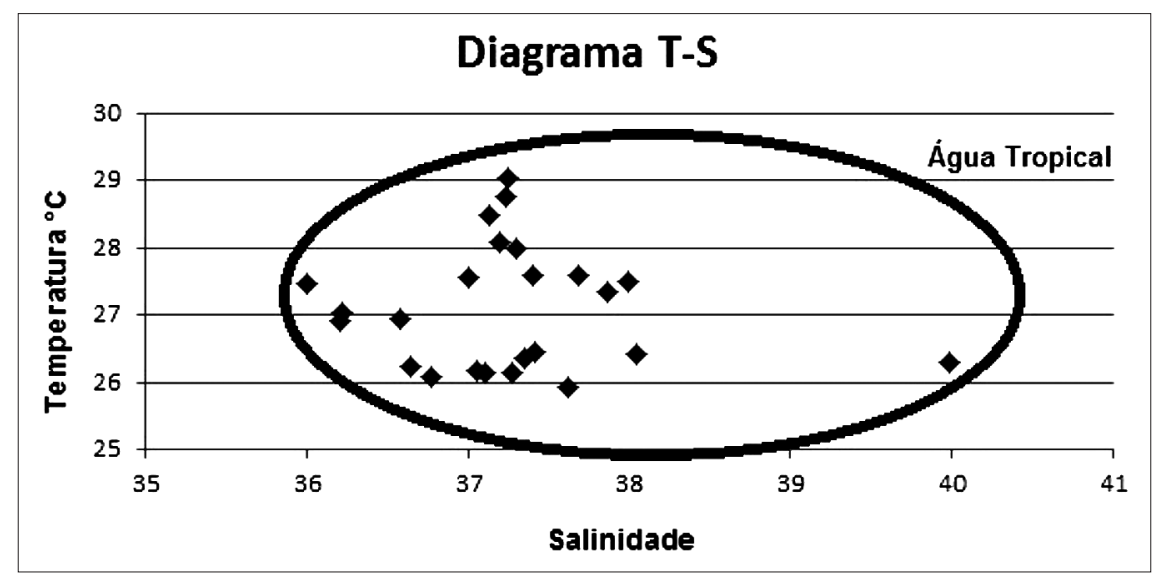

Figura 2 - Diagrama T-S para as estações de amostragem na plataforma continental de Salvador, Bahia, durante o período de abril de 2013 a janeiro de 2014

\section{Transparência}

O maior valor de transparência $(35 \mathrm{~m})$ foi registrado na campanha 1 no ponto BA4 e o menor $(5 \mathrm{~m})$ foi registrado na campanha 2 no ponto 1 . Não foi registrada variabilidade temporal $(\mathrm{p}>0,05)$, porém, foi registrada variabilidade espacial $(\mathrm{p}<0,05)$.

\section{Oxigênio dissolvido (OD)}

O menor valor de oxigênio dissolvido $\left(4,90 \mathrm{mg} . \mathrm{L}^{-1}\right)$ foi registrado no período de maior precipitação, campanha 3 ponto BA1, e o maior valor $\left(7,60 \mathrm{mg}\right.$. $\left.\mathrm{L}^{-1}\right)$ registrado na 
campanha 1 no ponto BA4. Foi registrada variabilidade temporal $(\mathrm{p}<0,05)$ e não foi registrada variabilidade espacial $(\mathrm{p}>0,05)$.

\section{Estrutura da Assembleia zooplantônica}

A análise qualitativa e quantitativa das amostras mostrou que a composição do zooplâncton foi característica de ambientes marinhos tropicais sendo compostas por organismos do holoplâncton e meroplâncton. Nas seis campanhas realizadas na transeção Salvador, entre a plataforma continental e o talude, foram identificados e quantificados 44 grupos do zooplâncton sendo registrados 6 filos (Protozoa, Cnidaria, Annelida, Mollusca, Echinodermata e Chaetognata) e 2 subfilos (Crustacea e Tunicata). Dentre os representantes do holoplâncton destacaram-se: Foraminifera, Gastropoda (veliger), Chaetognata, Cladocera, Ostracoda, Copepoda, Siphonophorae, Doliolidae, Salpidae e Larvacea) e do meroplâncton: Hydromedusae, Polychaeta, Bivalvia, Cirripedia, Decapoda, Stomatopoda, Porcellanidae, Brachyura, Ascidiacea, Amphipoda(Hyperiidea, Gammaridea) e Asteroidea.

Foram considerados característicos, os grupos que ocorreram em todas as campanhas, ou seja, onde a $\mathrm{FO}=100 \%$, totalizando 14 grupos, sendo eles: Shiphonophorae, Hydromedusae, Gastropoda (veliger), Polychaeta, Copepoda, Decapoda, Gammaridea, Brachyura, Chaetognata, Doliolidae, Salpidae, Larvacea e Radiolaria.

\section{Distribuição do zooplâncton}

Durante o período de amostragem, o holoplâncton predominou sobre o meroplâncton representando $83 \%$ da comunidade. A densidade total do zooplâncton $(629,66 \pm$ 1032,53 ) variou entre 12,5 org. $\mathrm{m}^{-3}$ e 4425 org. $\mathrm{m}^{-3}$ (Figura 3) e no geral, a densidade do macrozooplâncton apresentou um gradiente orientado no sentido costa-talude continental (Figura 4). Foi registrada variabilidade temporal $(\mathrm{p}<0,05)$ e não foi registrada variabilidade espacial $(\mathrm{p}>0,05)$. Copepoda foi o grupo que ocorreu em maior abundância, sendo registrados valores de densidade $(394,60 \pm 908,34)$ que variaram entre $2,81 \mathrm{org} / \mathrm{m}^{3}$ e 4143,75 $\mathrm{org} / \mathrm{m}^{3}$ (Figura 5). Foi registrado variabilidade temporal $(\mathrm{p}<0,05)$ e não foi registrado variabilidade espacial $(p>0,05)$.

\section{Riqueza}

Durante o período de amostragem a riqueza foi estimada através do cálculo do índice de riqueza de Margalef (IRM). A partir dos resultados obtidos foi possível observar que a riqueza variou entre 2,55 (mín) e 6,26 (máx). Não foi registrada variabilidade temporal $(\mathrm{p}<0.05)$ e apesar de também não ter sido registrada variabilidade espacial, a partir da riqueza média foi possível observar um gradiente decrescente orientado no sentido costa-oceano. (Figura 6).

\section{Relação entre a composição zooplanctônica e a estrutura oceanográfica}

A Análise de Correspondência Canônica não tendenciosa (DCCA) foi utilizada para investigar o tamanho do gradiente ambiental. Uma vez que este gradiente foi linear $(1,36)$, foi realizada uma Análise de Redundância (RDA) para verificar através do diagrama de ordenação, o principal padrão de variação na composição da assembleia zooplanctônica, em função da estrutura oceanográfica.

Os dois primeiros eixos canônicos da Análise de Redundância (AR) explicaram 88\% da variação percentual acumulada da relação entre composição do zooplâncton e a estrutura oceanográfica. A soma dos autovalores canônicos representou 32\% da inércia total, quando 
o ideal é superior a 20\%. Ou seja, nesta AR, 32\% da variabilidade na composição foi explicado pelo gradiente oceanográfico gerado pela massa de água.
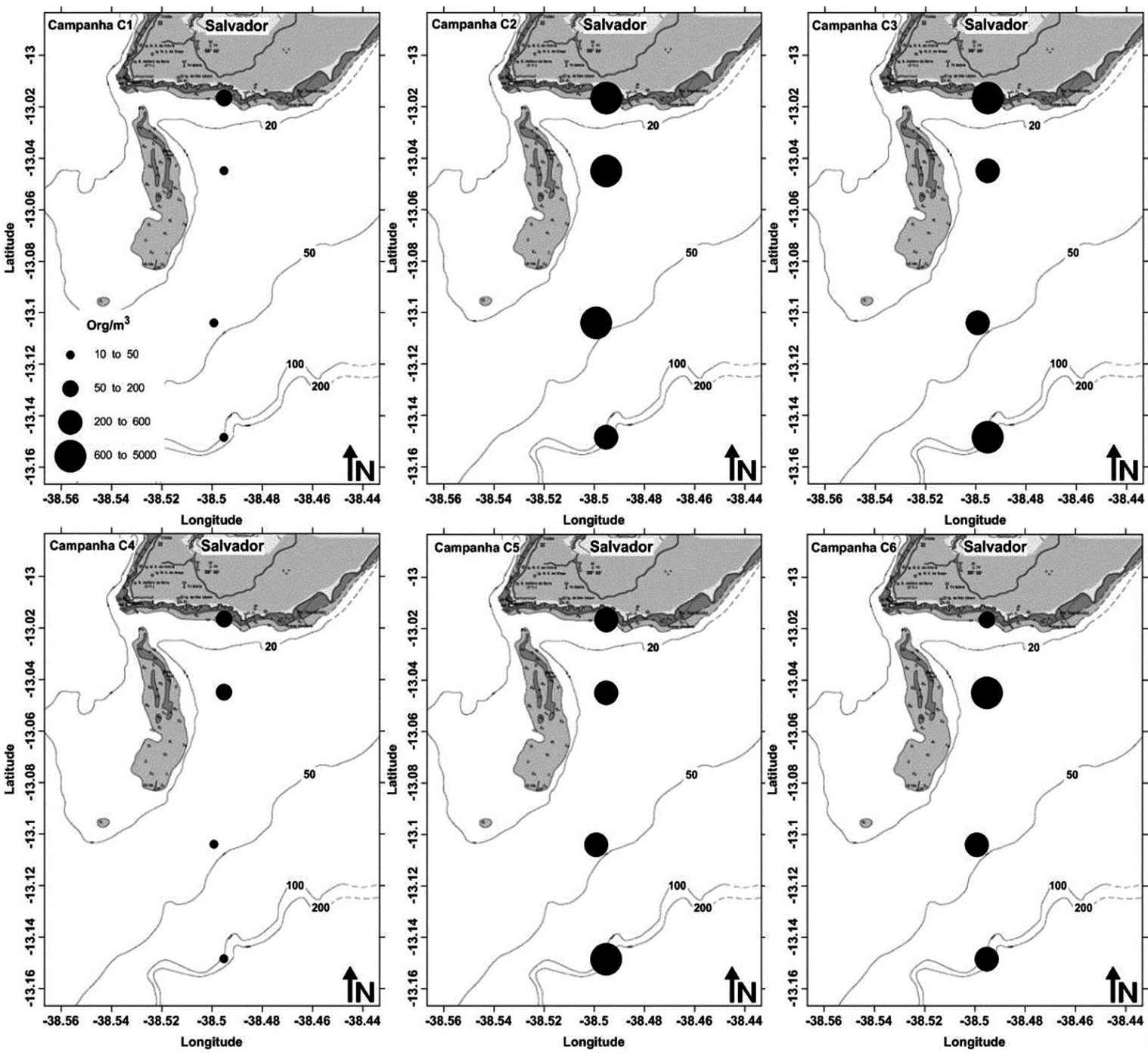

Figura 3 - Densidade total do zooplâncton obtida na plataforma continental de Salvador, Bahia, durante o período de abril de 2013 a janeiro de2014.

Figura 4 - Gradiente de densidade média do zooplâncton total na plataforma continental de Salvador, Bahia, durante o período de Abril de 2013 a Janeiro de2014.

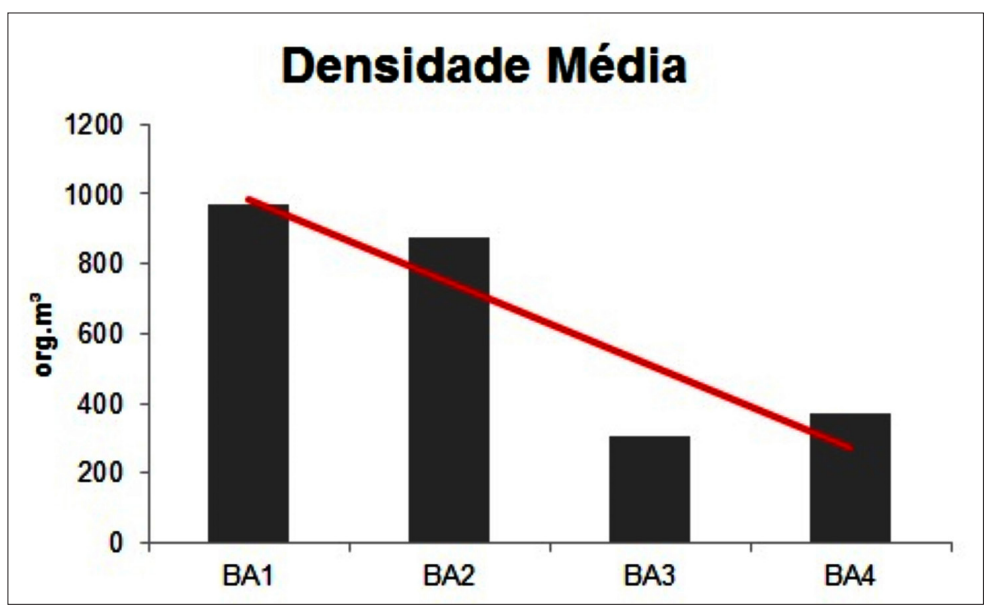



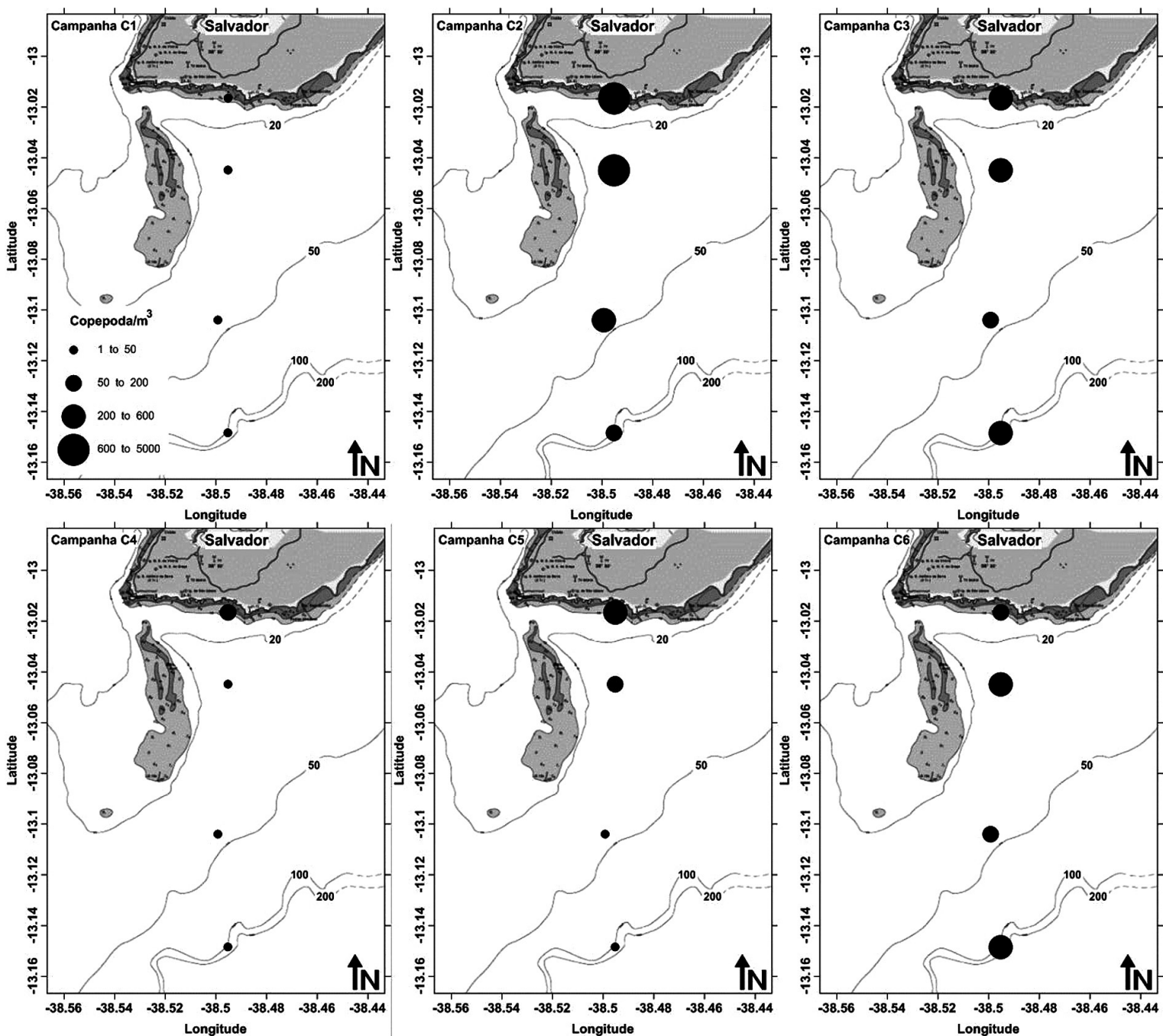

Figura 5 - Densidade de Copepoda obtida na plataforma continental de Salvador, Bahia, durante o período de abril de 2013 a janeiro de 2014

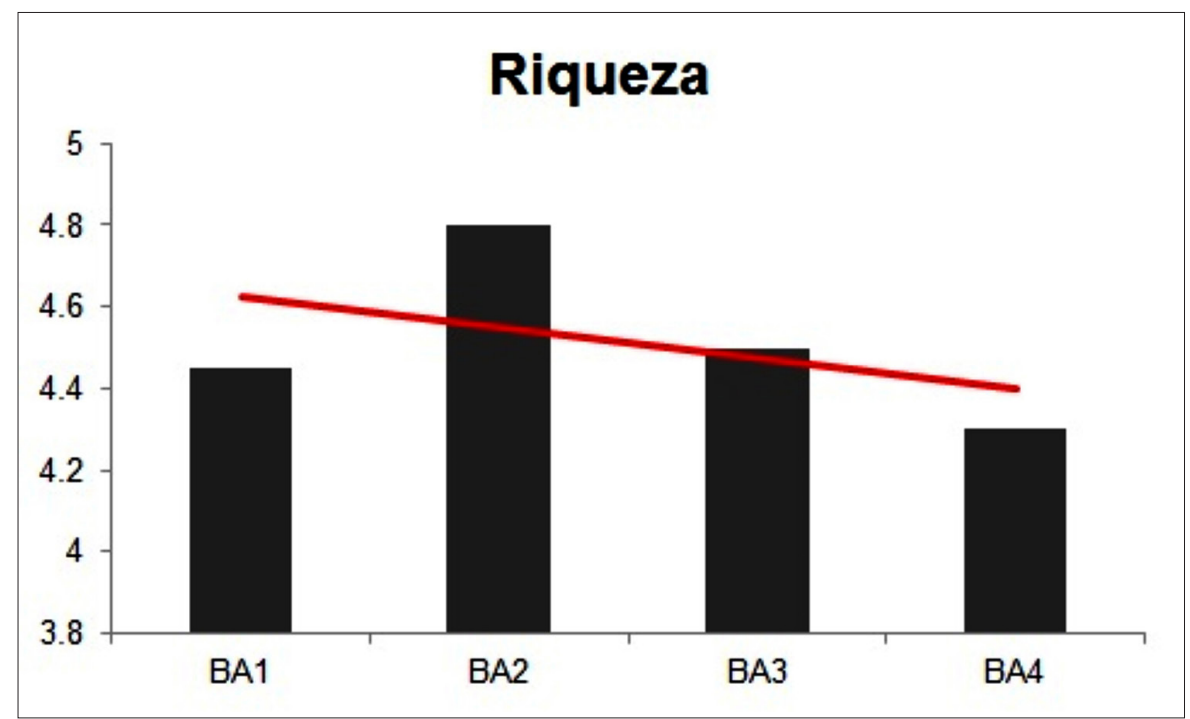

Figura 6 - Riqueza de Margalef obtida na plataforma continental de Salvador, Bahia, durante o período de abril de 2013 a janeiro de 2014 . 
Através do diagrama de ordenação obtido na RDA (Figura 7), foi possível verificar uma separação temporal entre as diferentes campanhas de amostragem em função das características oceanográficas e da composição do zooplâncton no ambiente marinho pelágico. Além disso a análise também mostrou uma clara variabilidade da densidade do zooplâncton relacionada às variáveis físicas e químicas (temperatura, salinidade, oxigênio dissolvido e transparência).

As campanhas 1, 4, 5 e 6, localizadas do lado esquerdo do diagrama apresentaram os maiores valores de temperatura, salinidade, oxigênio dissolvido e transparência e também estiveram associadas as menores densidades zooplanctônicas. Já as estações de amostragem, das campanhas 2 e 3 situadas no lado direito, apresentaram menores valores de temperatura, salinidade, oxigênio dissolvido e transparência e também as maiores densidades e grupos do zooplâncton.

A campanha 5 foi caracterizada pelo domínio de Gastropoda, a campanha 3 foi caracterizada pela ocorrência de Brachyura, Decapoda, Larvacea, Polychaeta, Salpidae e Siphonophorae. Já a campanha 2 foi dominada por: Copepoda, Doliolidae, Gammaridae, Hydromedusae e Radiolaria.

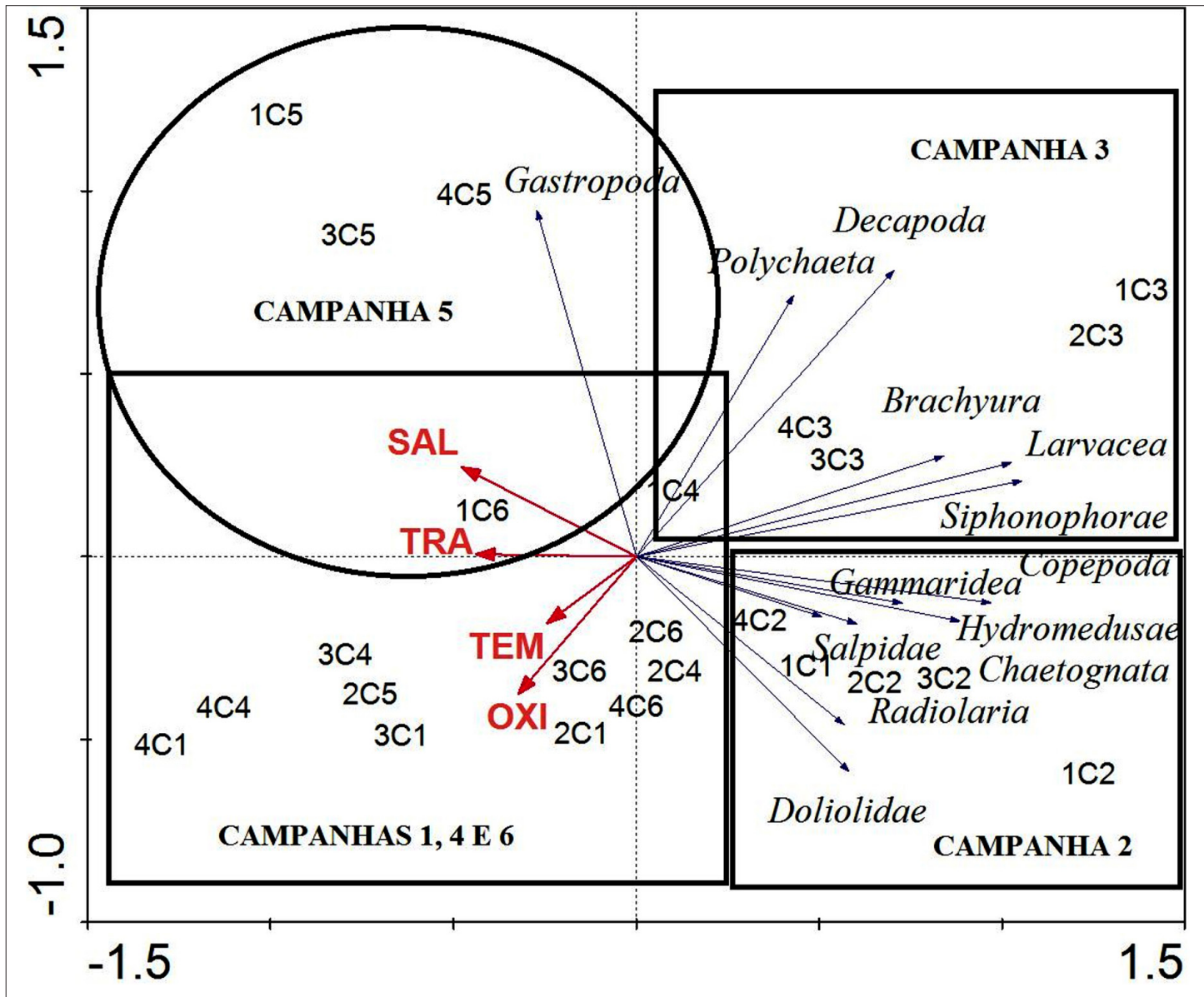

Figura 7 - Diagrama de Ordenação para a Análise de Redundância relacionando as estações de amostragem, a composição da assembléia zooplanctônica e a estrutura oceanográfica na plataforma continental de Salvador, Bahia durante o período de abril de 2013 a janeiro de 2014. (Estação 1: 1C1, 1C2, 1C3, 1C4, 1C5, 1C6; Estação 2: 2C1, 2C2, 2C3, 2C4, 2C5, 2C6; Estação 3: 3C1, 3C2, 3C3, 3C4, 3C5, 3C6; Estação 4: 4C1, 4C2, 4C3, 4C4, 4C5, 4C6; SAL, salinidade; TEM, temperatura; OXI, Oxigênio dissolvido; TRA, transparência). 


\section{DISCUSSÃO}

Entre abril de 2013 a janeiro de 2014, os valores de salinidade e temperatura indicaram a presença de uma única massa de água denominada Água Tropical que em função da estreita plataforma continental, com cerca de $10 \mathrm{~km}$ de extensão (Brandini et al., 1997; Mafalda et al.,2004; Dias et al., 2009), avança em direção a costa norte da Bahia atingindo a área de estudo.

Apesar do oxigênio dissolvido ter registrado seu menor valor na campanha 3 no ponto BA1 (4,9 mg. $\left.\mathrm{L}^{-1}\right)$, ainda esteve dentro do que é recomendado para águas salinas de classe tipo 2 e $3\left(>4,0 \mathrm{mg} . \mathrm{L}^{-1}\right)$, no qual se enquadra a área de estudo em questão, já que é utilizada para pesca amadora, recreação e navegação (CONAMA, 2005). Os maiores valores de oxigênio registrados na campanha 1 podem ser o resultado de uma intensa produtividade primária, como consequência de uma união de fatores como: 1. provável aumento na disponibilidade de nutrientes, já que a área de estudo está localizada numa região costeira onde o aporte de efluentes através de canais urbanos é comum; 2. Temperaturas maiores também registradas na campanha 1 e 3. elevada transparência também registrada nesse mesmo período. Esses fatores somados, podem ter favorecido a produção de oxigênio a partir da atividade fitoplanctônica já que a produtividade primária é limitada pela quantidade e intensidade de luz e nutrientes (Anastácio et al., 2012).

A transparência foi o único parâmetro que não variou significativamente entre as campanhas. Contudo foi verificado um gradiente espacial com um aumento ocorrendo à medida que se teve um afastamento da costa, corroborando com resultados obtidos por Mafalda Jr. et al. (2004). Em regiões costeiras a coluna d'água costuma registrar maior concentração de material particulado em suspensão resultante de uma maior turbulência, erosão e aportes oriundos da drenagem continental (Friedrich, 1969). Esse comportamento pode justificar o fato da transparência ter sido menor nos pontos mais costeiros (BA1 e BA2) e maior nas estações mais afastadas (BA3 e BA4).

Copépodes, quetognatos, cndiários e larváceos identificados na plataforma continetal de Salvador são típicos de ambiente marinho tropical (Neumann-Leitão et al., 1998; Mafalda, 2000; Mafalda et al., 2004).

Além disso, os organismos do holoplâncton representaram mais de $73 \%$ da abundância relativa total. Na região costeira do Ceará o mesozooplâncton da área adjacente ao Terminal Portuário do Pecém caracterizou-se pelo predomínio de espécies holoplanctônicas sobre espécies meroplanctônicas típicas de áreas costeiras representadas por copépodes, sifonóforos, sidromedusas e foraminíferos , (Garcia et al., 2007). Em estudo realizado no Golfo de Cádiz, resultados mostraram que mais de 98\% da abundância relativa total do zooplâncton foi representada por organismos do holoplâncton e apenas $2 \%$ por organismos do meroplâncton (Mafalda Jr. et al., 2007).

A densidade apresentou um gradiente orientado no sentido costa-talude com uma tendência dos maiores valores ocorrerem nas estações mais próximas à costa e os menores nas mais afastadas. Esse comportamento é comum às regiões marinhas tropicais e já foi registrado para outras regiões da costa brasileira (Lopes et al., 1999; Neumann-Leitão et al., 2008, Dias \& Bonecker 2009; Campos, 2014). Em ambientes marinhos tropicais é comum que a diversidade seja alta, acompanhada de baixas densidades/biomassa do zooplâncton (Neumann-Leitão et al., 2008).

A massa de Água Tropical, além de apresentar um caráter oligotrófico, suas características de temperatura e salinidade estiveram bem marcadas chegando a registrar valores 
médios acima do que é registrado na literatura para a região, segundo proposto por Mafalda et al. (2004). A pesar de em regiões marinhas tropicais a densidade do zooplâncton ser baixa, a densidade média do zooplâncton na plataforma continental de Salvador foi consideravelmente baixa, podendo esse comportamento estar associado às mudanças nas condições térmicas e salinas da massa de água. Na foz do Rio São Francisco NeumannLeitão et al. (1999) também registraram baixas densidades do microzooplâncton que variaram entre 468 e 3257 org. $\mathrm{m}^{-3}$ e para o mesozooplâncton valores entre 21 e 210 org.m- ${ }^{3}$.

Copepoda foi o grupo mais representativo já que ocorreu em todas as campanhas com uma densidade consideravelmente alta (com exceção da campanha 5, onde Gastropoda foi dominante, representando $76 \%$ da abundância relativa) assim como resultados obtidos por Dias \& Bonecker (2009) que chegaram a registrar densidades em torno de 7.473 ind. $\mathrm{m}^{-3}$

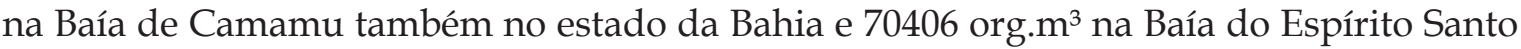
(Dias \& Bonecker, 2008). A dominância de Copepoda é uma característica comum às águas do Atlântico Tropical Sul (Neumann-Leitão et al.,1998; Neumann-Leitão et al., 2008) e este grupo costuma ser um grupo abundante nas águas costeiras e oceânicas, geralmente dominante nas assembleias mesozooplanctônicas tanto em termos de abundância, riqueza e biomassa além de representarem extrema importância na transferência de carbono nos oceanos (Berasategui et al., 2006; Wood-Walker et al., 2002).

Neves et al. (2003) apontaram que a estrutura da comunidade zooplanctônica depende Neves et al. (2003) apontaram que a estrutura da comunidade zooplanctônica depende da relação complexa de diversos fatores que incluem desde condições morfométricas e climáticas regionais a características físicas e químicas da água. Na baía de Camamu, a estreita plataforma permite a interação entre as comunidades costeiras e oceânicas que por sua vez são influenciadas pelos parâmetros ambientais de temperatura e salinidade, aqueles que caracterizam a estrutura da massa d'água (Dias et al., 2009).

Os valores de riqueza mostraram uma razoável biodiversidade visto que variaram entre 2,55 e 6,26, quando o limite mínimo para que a biodiversidade seja considerada alta é acima de 5 . O registro de 44 grupos do zooplâncton reforça tal diversidade. Estudos realizados em áreas costeiras e oceânicas como os de Neumann-Leitão, et al. (1999) identificaram 141 espécies mesozooplanctônicas e Cavalcanti \& Larrazábal (2004) registraram 63 espécies de macrozooplâncton na plataforma continental nordestina. Isso mostra que ambientes oceânicos embora oligotróficos, mantém uma alta diversidade e riqueza de espécies, às vezes maior que a encontrada em águas neríticas de alta produtividade (Díaz, 2007).

Através dos resultados obtidos na análise de ordenação é possível notar que os menores valores de densidade e riqueza do zooplâncton estão associados aos maiores valores de temperatura, salinidade, oxigênio dissolvido e transparência (campanha 1, 4, 5 e 6) e os maiores (campanha 2 e 3), associados aos menores respectivamente, reforçando o caráter oligotrófico intenso da massa de água tropical, sendo tal comportamento já observado na costa nordeste brasileira por Neumann-Leitão (1999) onde os menores valores de densidade corresponderam às águas oligotróficas presentes na região.

\section{REFERÊNCIAS BIBLIOGRÁFICAS}

Anastácio, R.S; Azeiteiro, U.M.M. \& Pereira, M.J.V. Climatic changes, nutrient imbalance and primary productivity in aquatic ecosystems. Trop. Ocean., Recife, v. 40, n. 1, p. 1-16. 2012. 
APHA. Standard Methods for the Examination of Water and Wastewater. $18^{\text {th }}$. Washington, D. C.: American Public Health Association, 1992.

Berasategui, A.D.; Menu Marque, S.; Gomez-Erache, M.; Ramírez, F.C.; Mianzan, H.W. \& Acha, E.M. Copepod assemblages in a highly complex hydrographic region. Estuar. Coast. Shelf Sci., v.66, p. 483-492. 2006.

Boltovskoy, D. Atlas Del Zooplacton Del Atlantico Sudoccidental: y métodos de trabajo com El zooplankton marino. INIDEP, 935p. Argentina, 1981.

Bonecker, S.LC.; Dias, C.O.; Fernandes, L.D.A. \& Ávila, L.R. M. In: VALENTIN J.L. 2006a. Características hidrobiológicas da região central da Zona Econômica Exclusiva brasileira (Salvador, BA, ao Cabo de São Tomé, RJ). Série Documentos REVIZEE - Score-Central. Ideal Gráfica e Editora Brasília, 2006.

Brandini, F.P.; Lopes, R.M.; Gutseit, K.S.; Spach, H.L. \& Sassi, R. Planctonologia na plataforma continental do Brasil: diagnose e revisão bibliográfica. MMA, CIRM, FEMAR,196 p. 1997.

Conselho Nacional de Meio Ambiente (CONAMA); Resolução n. 357 de 15 de março de 2005. Brasília, DF, 2005.

Campos, C.C. Assembleia de copépodes (Copepoda: Crustacea) na plataforma continental do nordeste do Brasil. 2014, 46p. Dissertação (Mestrado em Ciências Marinhas Tropicais). Instituto de Ciências do Mar, Universidade Federal do Ceará, Fortaleza, 2014.

Cooke, C.V.; Madureira, L.S.P.; Griep, G.H. \& Pinho, M.P. Análise de dados de ecossondagem de fundo oriundos de cruzeiros realizados entre Fortaleza (CE) e Chuí (RS) com enfoque na morfologia e tipos de fundo. Rev. Bras. Geofisica, Rio de Janeiro, v.25, n. 4, p. 443-457, 2007.

Dias, C.O.\& Bonecker, S.L.C. The Copepod Assemblage (Copepoda: Crustacea) on the Inner Continental Shelf Adjacent to Camamu Bay, Northeast Brazil. Zoologia, v. 26, n. 4, p. 629-40. 2009.

Dias, C.O. \& Bonecker, S.L.C. The Inter-Annual Variability of Planktonic Copepods in A Tropical Bay in Southeastern Brazil. Braz. Arch. Biol. Technol., v. 51, n. 4, p. 731-742. 2008.

Ekau, W. \& Knoppers, B. An introduction to the pelagic system of the north-east and east Brazilian shelf. Arch. Fish. Mar. Res., v. 47, n. 2-3, p. 113-132, 1999.

Friedrich, H. Marine Biology: Sidgwick \& Jackson, 472 p. London, 1969.

Garcia, T.M.; Lima, J.P. \& Castro Filho, R.S. Mesozooplâncton da região costeira próxima ao terminal portuário do Pecém - Estado do Ceará. Arq. Ciên. Mar, Fortaleza, v. 40, n. 2, p. $19-25.2007$

Garfield III, N. Brazil current at subtropical latitudes. Thesis (Doctor of Philosophy), University of Rhode Island, United States. 122p., 1990.

Hatje, V. \& Andrade, J.B. Baía de Todos os Santos: Aspectos oceanográficos. Salvador, EDUFBA, 306p. Salvador, 2009.

Keekler, D. SURFER for Windows. Version 6. User's Guide. 1995.

Kiørboe, T. A mechanism approach to plankton ecology. Princeton Universtity Press. Princeton, NJ. 2008.

Knoppers, B.; Ekau, W. \& Figueredo, A.G. The coast and shelf of east northeast Brazil and the material transport. Geo-Marine Letters. v.19, p. 171-178. 1999. 
Litchman, E.; Ohman, M.D. \& Kiørboe, T. Trait-based approaches to zooplankton communities. Jr. Plankton Res., v. 35, n. 3, p. 473-484. 2013.

Lopes, R M.; Brandini, F.P. \& Gaeta, S.A. Distribution patterns of epipelagic copepods off Rio de Janeiro (SE Brazil) in summer 1991/1992 and winter 1992. Hydrobiologia, v. 411, p. 161-174. 1999.

Mafalda-Junior, P.O.; Forte Neto, J.B.; Sallés, S.; Santos, C.; Souza, C.S.; Santos, J. J. \& Liralima, D. In: Hazin, F.H.V. Oceanografia Biológica: Biomassa Zooplanctônica na ZEE da Região Nordeste do Nordeste do Brasil. Coleção PROGRAMA REVIZEE SCORE NORDESTE. Martins \& Cordeiro Ltda, v. 2, p. 27-47. Fortaleza, 2009.

Mafalda-Junior, P.O.; Rubín, J.P. \& Souza, C.S. Mesozooplankton composition and distribution in relation to oceanographic conditions in the Gulf of Cádiz, Spain. Revista UDO Agrícola, v. 7, n. 1, p. 274-284, 2007.

Mafalda-Junior, P.O.; Sinque, C.; Brito, R.R.C. \& Santos, J. J. Biomassa planctônica, hidrografia e pluviosidade na costa norte da Bahia, Brasil. Trop. Ocean., Recife, v. 32, p. 143-158, 2004.

Neumann-Leitão, S.; Sant'anna, E.M.E.; Gusmão, L.M.O.; Nascimento-Vieira, D.A.; Paranaguá, M.N. \& Schwamborn, R. Diversity and distribution of the mesozooplankton in the tropical Southwestern Atlantic. J. Plankton Res., v. 30, n. 7, p. 795-805, 2008.

Neumann-Leitão S.; Souza, M.R.M.; Porto Neto, F.F.; Moura, M.C.O.; SILVA, A.P. \& Gusmão, L.M.O. Zooplâncton do estuário do rio São Francisco, Nordeste do Brasil. Trop. Ocean., v. 27, n. 1, p. 33-54,1999.

Neumann-Leitão, S.; Gusmão, L.M.O.; Nascimento Vieira, D.A.; Silva, T.A.; Silva, A.P.; Porto Neto, F.F. \& Moura, M.C.O. Biodiversidade do mesozooplâncton do nordeste do Brasil. Trop. Ocean., Recife, v. 26, n. 1, p. 27-34, 1988.

Neves, I.F.; Rocha, O.; Roche, K.F. \& Pinto, A.A. Zooplankton Community Structure of Two Marginal Lakes of the River Cuiabá (Mato Grosso, Brazil) with Analysis of Rotifera and Cladocera Diversity. Braz. J. Biol., São Paulo, v. 63, n. 2, p. 329-343, 2003.

Paredes, J.F. Evaluation of the environmental effects of the industrial effluents from Tibrás - Titânio do Brasil in the area under influence of its underwater outfalls. In: International Specialized Conference on Marine Disposal System, Lisboa. p. 105-125, 1991.

Pereira, P.M.S. A Sedimentação Areno-Lamosa do Baixo da Boca do Rio, Salvador, Bahia. Monografia. Universidade Federal da Bahia, 63p. Salvador, 2009.

Pereira, A.G.V. \& Lessa, G.C. Varying patterns of water circulation in canal de Cotegipe. Rev. Bras. Geofísica, Rio de Janeiro, v.27, n. 1, p. 103-119, 2009.

Ter Braak, C.J.F. Canonical correspondence analysis: a new eigenvector technique for multivariate gradient analysis. Ecology. v. 67, n. 5, p. 1167-1179, 1986

Ter Braak, C.J. F. \& Smilauer, P. CANOCO Reference Manual User's Guide to Canoco for Windows Microcomputer Power. p. 352, 1998

Thompson, G.A.; Dinofrio, E.O. \& Alder, V.A. Structure, abundance and biomass size spectra of copepods and other zooplankton communities in upper waters of the Southwestern Atlantic Ocean during summer. J. Plankton Res., v. 35, n. 3, p 610-629, 2013.

Woodd-Walker, R.S.; Ward, P. \& Clarke, A. Large-scale patterns in diversity and community structure of surface water copepods from the Atlantic Ocean. Mar. Ecol. Prog. Ser., v. 236, p. 189-203, 2002. 